\title{
Interrelation between legibility attributes and park utilization as determinants for responsive neighborhood parks
}

\begin{abstract}
ABSTRCT
The rising need for social interaction is evident particularly in urban areas due to a stressful urban life and social isolation. In residential areas, neighborhood parks have an important function in supporting social integration and social bonding. Nevertheless, studies have indicated that these parks are not fully utilized for the benefits of residents which could be due to the lack of parks' legibility. This paper investigates the interrelationship between park utilization and legibility of three neighborhood parks within new residential developments in Malaysia. The research findings are based on field observations and a questionnaire survey conducted with 387 residents of the areas. Results indicate a significant correlation between park utilization and park legibility, implying the potential for social interactions among residents within a legible park setting. The level of the park visual obstruction and the clarity of its structure were found to be strongly linked with park utilization and the intensity of outdoor activities. However, certain critical physical characteristics including the critical density of population and gated neighborhoods may decrease the positive effect of park's legibility. The findings inform the crucial role of legible environment in the design and planning of neighborhood parks to afford socially responsive public spaces thus enhances the social life of urban dwellers.
\end{abstract}

Keyword: Park utilization; Social integration; Legibility; Neighborhood parks; Quality of life. 